\title{
Direction detection thresholds of passive self-motion in artistic gymnasts
}

\author{
Matthias Hartmann · Katia Haller · Ivan Moser • \\ Ernst-Joachim Hossner $\cdot$ Fred W. Mast
}

Received: 2 May 2013 / Accepted: 10 January 2014 / Published online: 25 January 2014

(C) Springer-Verlag Berlin Heidelberg 2014

\begin{abstract}
In this study, we compared direction detection thresholds of passive self-motion in the dark between artistic gymnasts and controls. Twenty-four professional female artistic gymnasts (ranging from 7 to 20 years) and agematched controls were seated on a motion platform and asked to discriminate the direction of angular (yaw, pitch, roll) and linear (leftward-rightward) motion. Gymnasts showed lower thresholds for the linear leftward-rightward motion. Interestingly, there was no difference for the angular motions. These results show that the outstanding selfmotion abilities in artistic gymnasts are not related to an overall higher sensitivity in self-motion perception. With respect to vestibular processing, our results suggest that gymnastic expertise is exclusively linked to superior interpretation of otolith signals when no change in canal signals is present. In addition, thresholds were overall lower for the older (14-20 years) than for the younger (7-13 years) participants, indicating the maturation of vestibular sensitivity from childhood to adolescence.
\end{abstract}

Keywords Self-motion perception - Self-motion thresholds · Vestibular processing - Sport expertise . Gymnastics · Vestibular plasticity · Vestibular aging

M. Hartmann $(\bowtie) \cdot$ I. Moser $\cdot$ F. W. Mast

Department of Psychology, University of Bern, Fabrikstrasse 8, 3000 Bern, Switzerland

e-mail: matthias.hartmann@psy.unibe.ch

M. Hartmann · F. W. Mast

Center for Cognition, Learning and Memory, University of Bern,

Bern, Switzerland

K. Haller · E.-J. Hossner

Department of Sport Science, University of Bern,

Bern, Switzerland

\section{Introduction}

The vestibular system, located in the inner ear, provides the brain with information about the direction and magnitude of angular motion (encoded by the semicircular canals) as well as the direction and magnitude of linear accelerations, including changes in body orientation with respect to gravity (encoded by the otolith organs). Thus, vestibular processing plays a crucial role in the control of body posture and body motion. These functions are not only important in everyday life but also in professional sports such as artistic gymnastics. Artistic gymnastics is a highly demanding sport that includes complex body motions performed on the floor as well as on bars or balance beams. For each action, an exact control of body posture is crucial (e.g., Louer et al. 2012). Indisputably, the sport-specific skill level as well as factors such as muscular strength, flexibility, and coordination distinguishes high-performance gymnasts from the normal population. In this study, we investigated whether the perception of self-motion is different in artistic gymnasts when compared to controls.

To date, the impact of gymnastics expertise on the perception of self-motion, such as vestibular perception, is not clear. On the one hand, it has been shown that gymnasts outperform non-gymnasts in balance and postural control (e.g., Carrick et al. 2007; Gautier et al. 2008; Hrysomallis 2011) and in the perception of body orientation (Bringoux et al. 2000). Based on these results, one could assume that artistic gymnasts have developed an increased sensitivity for vestibular information (Bringoux et al. 2000). On the other hand, performing gymnastics strongly stimulates the vestibular system, which could lead to habituation of vestibular responses. Vestibular habituation has been described at the level of the vestibulo-ocular reflex (VOR; see Blair and Gavin 1979; Clément et al. 2008; Jäger and 
Henn 1981). The VOR is defined by rapid compensatory eye movements opposite to the direction of head rotation, enabling visual stability of the external world during head movements. A decrease in the velocity of the VOR has been found in artistic gymnasts (Quarck and Denise 2005), ice skaters (Alpini et al. 2009; Tanguy et al. 2008a, b), and ballet dancers (Osterhammel et al. 1968; Tschiassny 1957). It has been concluded that VOR modulation reflects vestibular habituation as a consequence of the repeated exposure to angular acceleration during exercise (e.g., Quarck and Denise 2005). However, some studies also found opposite effects. For example, Lee et al. (2004) found an increase in the velocity of compensatory eye movements as a result of flight training in pilots. Thus, the exact nature of these VOR modulations remains controversial. It is not clear whether these modulations are driven by motor or sensory processes and, more specifically, to what extent the vestibular part of the visual-vestibular network is influenced by expertise, since the visual system was involved during training and VOR assessment.

Recently, we found that passive self-motion perception can be improved via perceptual training in the light, but the training had no influence on self-motion perception in the dark (Hartmann et al. 2013). These results suggest that improvements in self-motion perception were driven by an alteration in those parts of the network processing visual information rather than the vestibular parts of the network alone. However, the near-threshold motion stimuli used in this perceptual training cannot be compared with the powerful long-time stimulation of the vestibular system during gymnastic training. Therefore, it remains an open question whether vestibular thresholds can be improved by a more extensive training of self-motion.

To address this question, we compared self-motion discrimination thresholds of artistic gymnasts and controls in the dark. Participants were passively displaced by means of a motion platform and asked to indicate the direction of motion. These thresholds can be regarded as indicator of vestibular sensitivity (Benson et al. 1986; Bertolini et al. 2012; Kingma 2005; Valko et al. 2012; MacNeilage et al. 2010).

Participants were asked to discriminate roll, pitch, and yaw rotations as well as leftward-rightward translations (y, see Fig. 1). Roll and pitch rotations coactivate the semicircular canals and the otolith organs, and are part of most (if not all) gymnastic movements (Bechter et al. 2009). Therefore, we expected the largest difference between artistic gymnasts and controls for these thresholds. Yaw (semicircular canals) and linear leftward-rightward motions (otoliths) lead to isolated activations of either vestibular subsystem (Merfeld 2012). These motions occur less often during gymnastic training, and we therefore expect smaller differences for these thresholds. Moreover, we assessed two

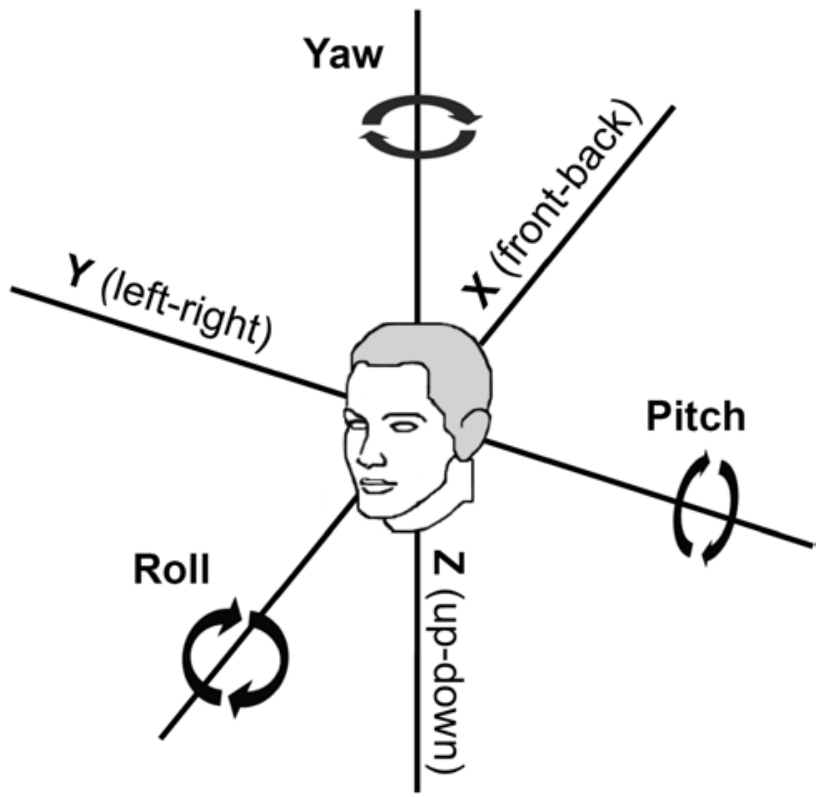

Fig. 1 Coordinate system with the linear $(x, y, z)$ and angular (yaw, pitch, roll) motion directions

thresholds for each of the four different types of motion: once with a short motion duration $(0.5 \mathrm{~s})$ and once with a longer motion duration $(3 \mathrm{~s})$. Previous studies showed that self-motion perception thresholds depend on the duration of motion (Grabherr et al. 2008). Given that slow motions are less typical for gymnastic motion sequences, we expected that differences between artistic gymnasts and controls would be more evident for the shorter motion durations.

To conclude, this study aims to evaluate whether artistic gymnasts differ from controls in self-motion perception. Moreover, this study was motivated by potential applications. If self-motion perception thresholds are lower in artistic gymnasts, an adapted version of our paradigm could be used as a tool to support the evaluation of young talents or to develop a novel training approach. Furthermore, this is to the best of our knowledge the first study investigating self-motion perception thresholds in children and young adults. Previous studies with older participants (between 20 and 70 years) found an increase in thresholds as a function of age (Kingma 2005; Roditi and Crane 2012a, b). Here, we are able to further investigate the relationship between age and self-motion perception in a young sample.

\section{Method}

Participants

Forty-eight participants were recruited for this study. Half of the participants were artistic gymnasts (mean age: 13.2, 
Fig. 2 Acceleration, velocity, and displacement as a funcmotion durations $3 \mathrm{~s}$ (upper panel) and $0.5 \mathrm{~s}$ (lower panel) tion of time for the two angular
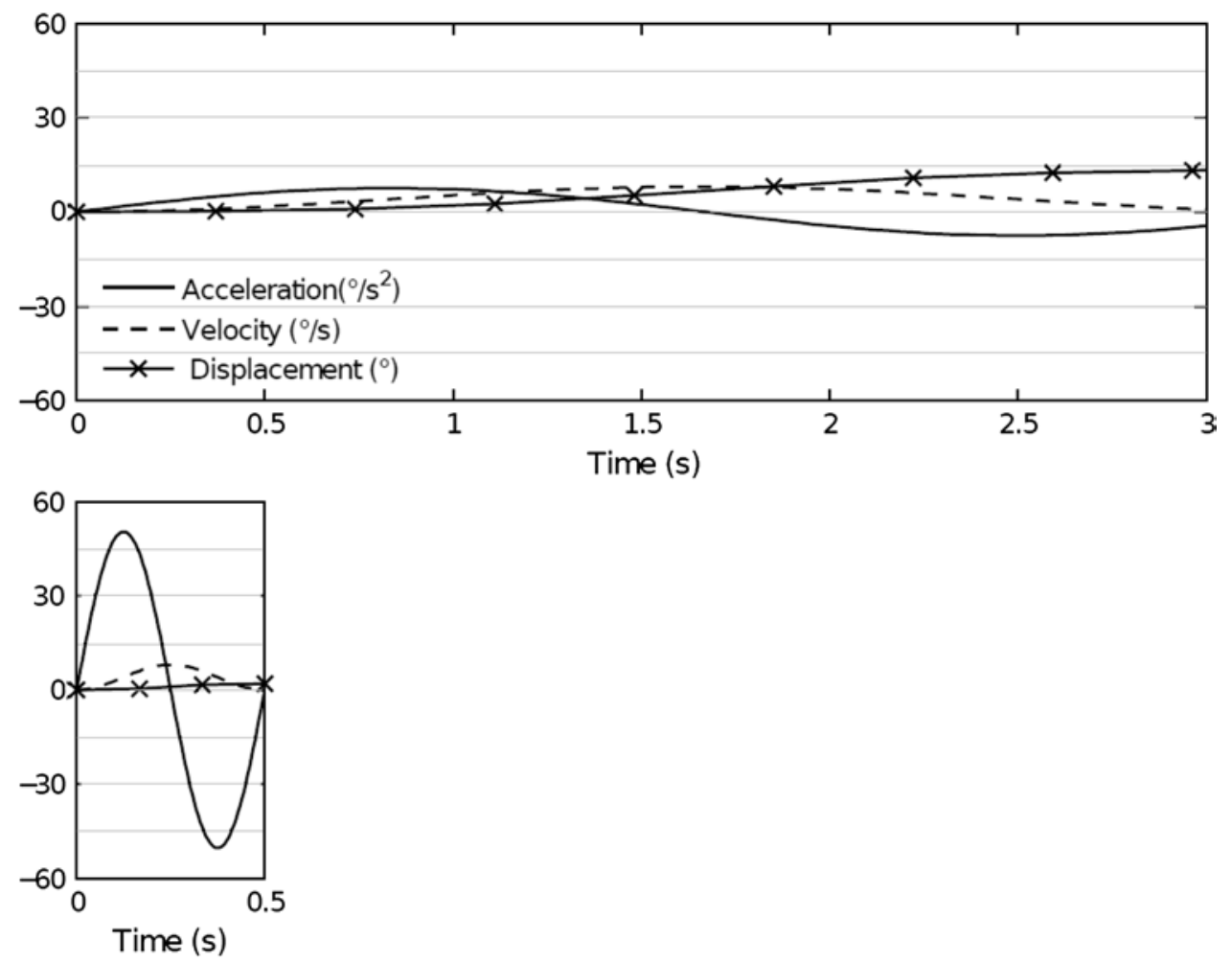

ranging from 7 to 19 , all female) and the other half served as control group (mean age: 13.9, ranging from 7 to 20, all female). The artistic gymnasts trained on average for $21.4 \mathrm{~h}$ per week (SEM $=0.96)$, and the average year of training was $8.6(\mathrm{SEM}=0.79)$. They were recruited from the regional $(n=18)$ and national $(n=6)$ elite sport center for artistic gymnasts in Bern/Switzerland and Magglingen/ Switzerland, respectively. The gymnasts' sample included National and European champions in various disciplines in artistic gymnastics. None of the participants in the control group was engaged in sport activity of more than $4 \mathrm{~h}$ per week. All participants completed a questionnaire to assess health and possible vestibular-related disorders such as dizziness, vertigo, or neurological issues. None of them indicated any history of vestibular disorder. The study was approved by the local Ethics Committee. All participants gave informed consent prior to the study (respectively, their parents when participants were under 16 years, following the guidelines of the local Ethics Committee).

\section{Apparatus and motion stimuli}

Motion stimuli were generated by means of a 6-degree-offreedom electric motion platform (6DOF2000E, MOOG Inc., East Aurora, NY; see Fig. 1 in Hartmann et al. 2012, for an image of the apparatus). We used single-cycle sinusoidal acceleration motion profiles (Grabherr et al. 2008). We tested pitch, roll, yaw, and y translation motion stimuli (see Fig. 1). The duration of the motion stimuli was 0.5 or 3 s. See Fig. 2 for acceleration, velocity, and position parameters for each motion duration. A PC with a Matlab-based custom-made software was used to control the motion platform and to record participants' responses. Statistical analysis was performed with IBM SPSS Statistics 19.

\section{Procedure}

Participants were seated in a chair that was mounted on the motion platform. Seat belts were fastened around participants' shoulders, torso, and hips. Their heads were restrained with a helmet and fixation straps. An adjustable foot rest served to position the feet. To minimize a posself-motion perception, participants were blindfolded and exposed to white noise presented via in-ear headphones.

Eight different self-motion velocity thresholds were recorded in separate blocks (roll, pitch, yaw, and y translation, each 0.5 and $3 \mathrm{~s}$ ). Participants were asked to press either the button in their left or right hand, according to the instructions provided before each block. For y translation and yaw and roll rotation, participants were asked to press the left button when they experienced a leftward motion and the right button when they experienced a rightward motion. For pitch rotation, they were asked to press the right button when they experienced a forward sibly confounding influence of visual or auditory cues on 
rotation and the left button when they experienced a backward rotation (the experimenter made clear what forward and backward rotation means by demonstrating a forward and backward bow). The participants were asked to respond after the motion had stopped. In case the participants were uncertain about the direction of self-motion, they were instructed to make their best guess. No feedback was provided. The order of the different types of motions was counterbalanced across participants. The blocks with the two different durations of the same motion were never presented in succession. The blocks were separated by a short break in which the lights were switched on. After the fourth block, a longer break was provided (10 $\mathrm{min})$ where participants came out of the chair in order to relax and diminish discomfort and fatigue. A complete experimental session (including debriefing) lasted about $1 \mathrm{~h}$ and $45 \mathrm{~min}$. Thresholds were determined by means of a two-alternative 3-down 1-up adaptive staircase procedure following a PEST algorithm (Leek 2001; Taylor and Creelman 1967). In this procedure, three correct responses in a row are needed in order for the velocity level to be reduced. The velocity level is increased after each incorrect response. The initial peak velocity at the beginning of the procedure was well above threshold (starting velocity values were $8 \%$ for angular motion and $0.06 \mathrm{~m} / \mathrm{s}$ for linear motion, which equals to accelerations of $0.38 \mathrm{~m} / \mathrm{s}^{2}$ for $0.5 \mathrm{~s}$ and to $0.06 \mathrm{~m} / \mathrm{s}^{2}$ for $3 \mathrm{~s}$ y translation stimuli). After three correct responses, the velocity was reduced by the factor 2 (e.g., from 8 to $4 \%$ s for angular motions). As soon as participants give an incorrect response, the factor according to which the velocity of the next motion is raised (or reduced again after three further correct responses in a row) changes according to the algorithm described in Taylor and Creelman (1967). The procedure stopped when five maximum reversals and six minimum reversals were reached. A maximum reversal is defined by three correct responses in a row after an incorrect response. A minimum reversal means an incorrect response following at least three correct responses. The last minimum reversal was reached on average after 64 trials. See Fig. 3 for an example of a staircase history.

During the staircase procedure, the motion platform was not repositioned after each trial. Motion stimuli were generated online by a custom-made software (based on Matlab). The direction of each motion was chosen randomly by the software. However, the direction of motion sometimes needed to be reversed by the experimenter in order to account for the displacement limits of the device. Moreover, the experimenter controlled for the motion directions of the initial trials in such a way that the participants' position was upright and centered when motion intensities reached a near-threshold level (around $1^{\circ}$ for angular motion).

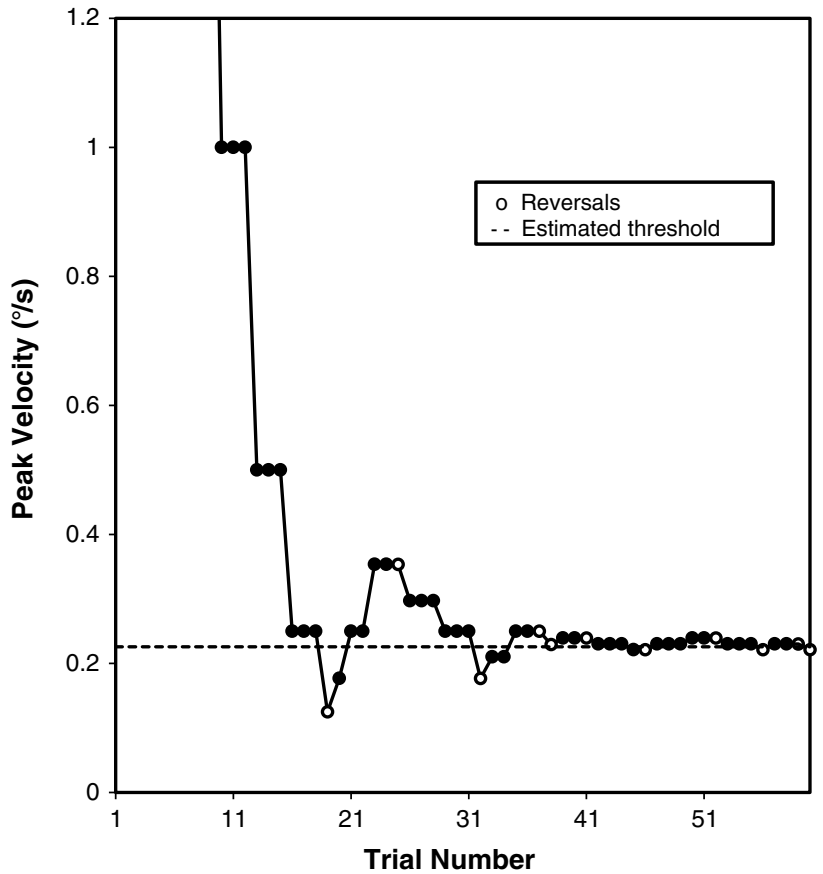

Fig. 3 Example of a 3-down, 1-up staircase procedure of a participant for 0.5 -s pitch rotation. Each motion stimulus is indicated by a circle. White circles represent reversals that are defined as incorrect response after three correct responses (minimum reversal) or as the third correct response in a row after an in correct response. The dotted line represents the estimated threshold, defined as the mean of the last minimum and maximum reversal. Initial peak velocity was $(8 \% \mathrm{~s})$, well above threshold

Each motion stimulus was preceded by a low-pitched tone (2,000 ms before motion onset). A high-pitched tone was played $500 \mathrm{~ms}$ after motion offset in order to indicate that the motion had stopped and to prompt the participants to respond. When the motion had stopped and the response was given, the next trial was triggered by the experimenter with a delay of approximately $3 \mathrm{~s}$. This resulted in an interval between motion stimuli of at least $6.5 \mathrm{~s}$ : $0.5 \mathrm{~ms}$ duration of the offset-motion-tone + at least $1 \mathrm{~s}$ until response was given (non-speeded responses) +3 s delay between response and triggering of the new trial $+2 \mathrm{~s}$ low-pitched tone before motion onset of the next motion stimulus.

\section{Data analysis}

Self-motion direction detection thresholds were computed by averaging the peak velocity of the last minimum and the last maximum reversal (see, e.g., Hartmann et al. 2013; Grabherr et al. 2008). This procedure results in a threshold value of $79.4 \%$ correctly detected trials.

Due to technical problems, six datasets were lost $(1.6 \%)$. The remaining thresholds were log-transformed to normalize their distribution (Berry 1987; Keene 2007). Threshold 
Table 1 Mean self-motion perception thresholds for each type and duration of motion

The age range of younger participants was $8-13$ and that of older participants $14-19$

\begin{tabular}{|c|c|c|c|c|c|c|}
\hline \multirow[t]{2}{*}{ Duration } & \multicolumn{2}{|c|}{ Younger participants } & \multicolumn{2}{|c|}{ Older participants } & \multicolumn{2}{|c|}{ All participants } \\
\hline & $0.5 \mathrm{~s}$ & $3 \mathrm{~s}$ & $0.5 \mathrm{~s}$ & $3 \mathrm{~s}$ & $0.5 \mathrm{~s}$ & $3 \mathrm{~s}$ \\
\hline Pitch $(\% / s)$ & 0.38 & 0.72 & 0.28 & 0.52 & 0.33 & 0.61 \\
\hline Roll (\%) & 0.40 & 0.65 & 0.22 & 0.43 & 0.30 & 0.53 \\
\hline Yaw $(\%$ s) & 1.27 & 1.09 & 0.54 & 1.16 & 0.83 & 1.12 \\
\hline $\mathrm{Y}\left(\mathrm{m} / \mathrm{s}^{2}\right)$ & 0.05 & 0.03 & 0.03 & 0.02 & 0.04 & 0.03 \\
\hline
\end{tabular}

data from five out of the eight motion conditions violated normal distribution, as revealed by the KolmogorovSmirnov test $(p<0.05)$. After log transformation, normal distribution was no longer violated (for all $p>0.229$ ).

In order to investigate possible effects of age and to account for different amount of training years for older and younger gymnasts, we created the variable age (younger, older) based on the median split (13 years). The mean age of the younger group was 10.1 and that of the older group 16.9. For the gymnasts, the mean years of training in the younger group was 5.2 and in the older group 12.0.

The four different types of motion (pitch, roll, yaw, and $\mathrm{y}$ translation) were analyzed by means of separate repeated measures analysis of variance (ANOVA) with the betweenvariables group (gymnasts vs. controls), age (younger vs. older), and the within-variable duration of motion (500 vs. $2,000 \mathrm{~ms}$ ). The ANOVAs were performed on the log thresholds. For the sake of clarity, we report non-log means. All reported means are back-transformed log means. Thresholds for the angular motions (pitch, roll, and yaw) are expressed in $\%$, whereas thresholds for the linear motion (y) are expressed in $\mathrm{m} / \mathrm{s}^{2}$.

\section{Results and discussion}

Mean thresholds for each type and duration of motion are summarized in Table 1. Moreover, mean threshold of the gymnasts and controls for each type of motion for the younger and older group is presented in Fig. 4. Statistical results for the four different types of motion are presented in Table 2. The most important findings are summarized below.

\section{Pitch rotation}

Most importantly, there was neither a main effect of group nor any interaction with group. Notably, however, the interaction between group and age was nearly significant $(p=0.052)$. For the older group, thresholds tended to be lower for gymnasts $(M=0.35$, SEM $=0.10)$ when compared to controls $(M=0.43$, SEM $=0.09)$, while the opposite was true for the younger group (see Fig. 4). Moreover, there was a main effect of age: Thresholds of the older
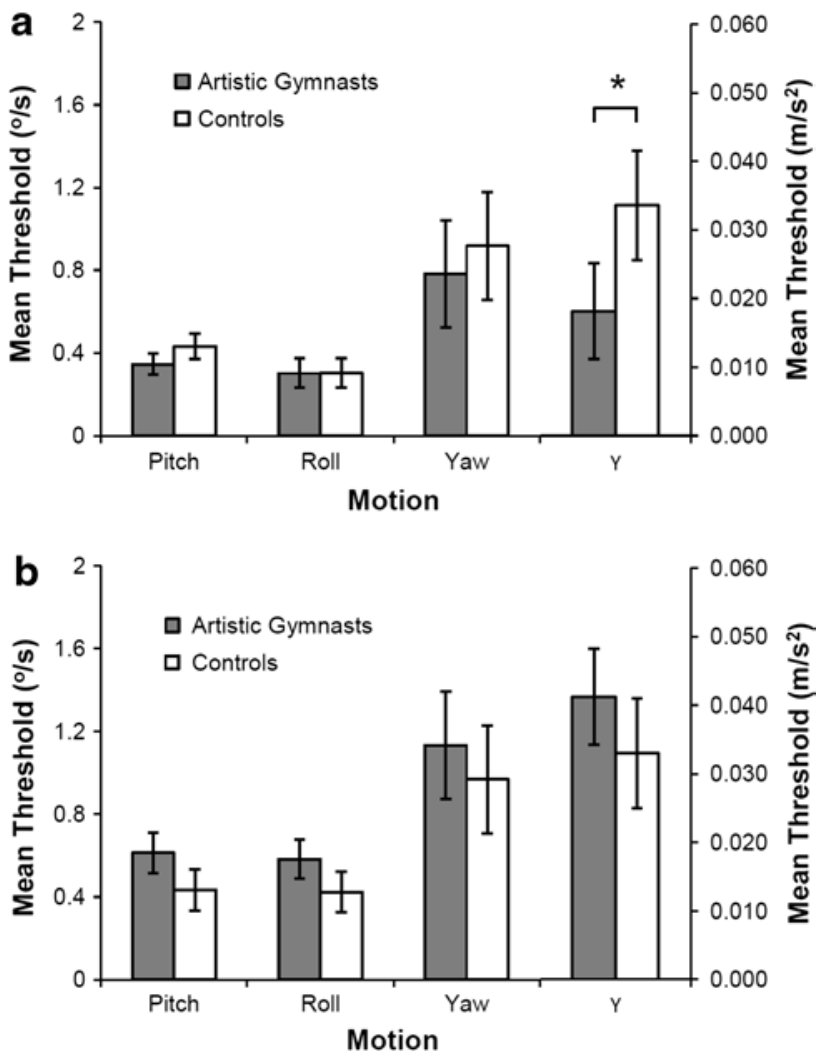

Fig. 4 Mean direction detection thresholds of passive self-motion for the older (a) and younger (b) artistic gymnasts and controls for the four different motions (data averaged over 0.5-s and 3-s motion). Pitch, roll, and yaw thresholds are expressed in velocity units $(\%)$ and $y$ translation thresholds are expressed in acceleration units $\left(\mathrm{m} / \mathrm{s}^{2}\right)$. The asterisk indicates a significant difference between gymnasts and controls for the linear leftward-rightward motion (y). Error bars depict \pm 1 SEM

group $(M=0.38, \mathrm{SEM}=0.07)$ were lower when compared to the younger group $(M=0.52, \mathrm{SEM}=0.07)$. There was also a main effect of duration of motion: Thresholds for the shorter motion $(M=0.33$, SEM $=0.08)$ were lower when compared to the longer motion $(M=0.61, \mathrm{SEM}=0.04)$.

\section{Roll rotation}

Most importantly, there was neither a main effect of group nor any interaction with group (see Table 2). There 
Table 2 Results of the analysis of variance with the variables group, age, and duration of motion

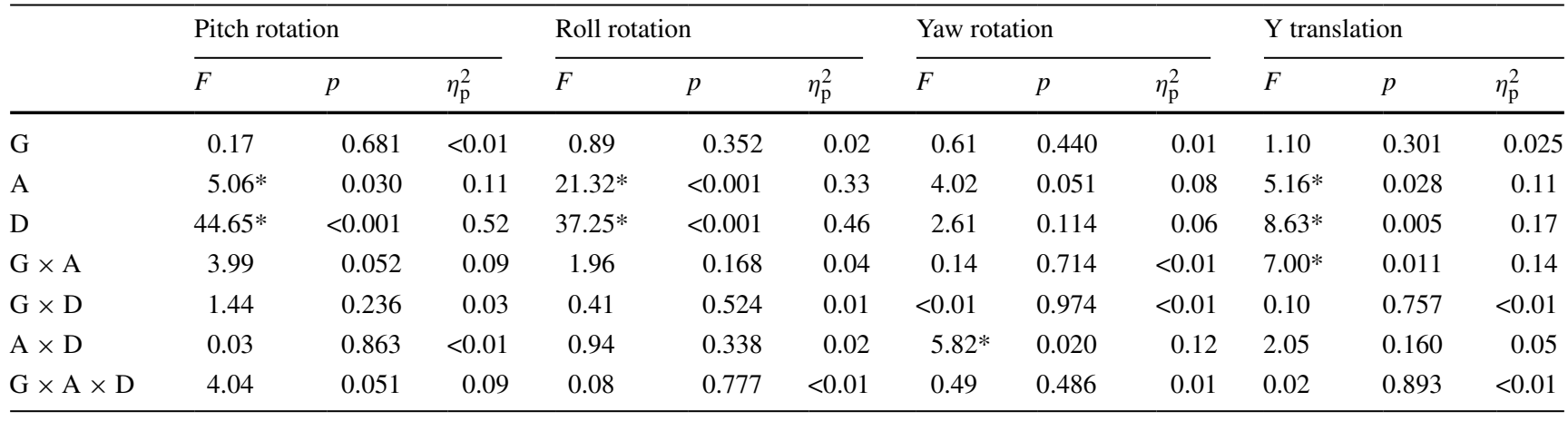

Dependent variables for pitch, roll, and yaw rotation were velocity thresholds $(\% / \mathrm{s})$ and for $\mathrm{y}$ translation acceleration thresholds in $\left(\mathrm{m} / \mathrm{s}^{2}\right)$

$\mathrm{G}=$ group (gymnasts vs. controls), $\mathrm{A}=$ age, $\mathrm{D}=$ duration of motion $(0.5 \mathrm{~s}$ vs. $3 \mathrm{~s})$

* Significant $F$ values $(p<0.05)$

was a main effect of age: Thresholds of the older group $(M=0.31$, SEM $=0.05)$ were lower when compared to the younger group $(M=0.51, \mathrm{SEM}=0.05)$. There was also a main effect of duration of motion: Thresholds for the shorter motion $(M=0.30, \mathrm{SEM}=0.03)$ were lower when compared to the longer motion $(M=0.53$, SEM $=0.06)$.

\section{Yaw rotation}

As for pitch and roll, there was neither a main effect of group nor any interaction with group (see Table 2). Unlike roll and pitch, there was no main effect of duration of motion, but duration of motion interacted with age. Bonferroni post hoc tests revealed significant lower thresholds for the shorter motion $(M=0.54, \mathrm{SEM}=0.19)$ when compared to the longer motion $(M=1.16, \mathrm{SEM}=0.22)$ for the older group only. Moreover, the main effect of age nearly reached significance $(p=0.051)$ : Thresholds of the older group $(M=0.79, \mathrm{SEM}=0.18)$ tended to be lower when compared to the younger group $(M=1.18, \mathrm{SEM}=0.18)$.

\section{Y translation}

There was no main effect of group, but group interacted with age (see Table 2). For the older group, Bonferroni post hoc tests revealed significant lower thresholds for gymnasts $(M=0.018$, SEM $=0.007)$ when compared to controls $(M=0.034$, SEM $=0.008)$. No difference between gymnasts and controls was found for the younger group $(p=0.530)$. Moreover, there was a main effect of age: Thresholds of the older group $(M=0.024$, SEM $=0.005)$ were lower when compared to the younger group $(M=0.036, \mathrm{SEM}=0.005)$. There was also a main effect of duration of motion: Thresholds for the shorter motion ( $M=0.036$, SEM $=0.006)$ were higher when compared to the longer motion $(M=0.025$, SEM $=0.003)$.
Vestibular thresholds

In general, self-motion perception thresholds reported in this study yielded comparable values to those found in other studies. For example, yaw rotation thresholds reported in Grabherr et al. (2008) for 2-s motion duration was around $0.5 \% \mathrm{~s}$ (the mean of the older group in this study was $0.54 \%$ ). Similarly, Grabherr et al. report yaw rotation thresholds between 1.5 and $2 \%$ for 5 -s motion duration, while the mean yaw rotation threshold in our study for the older group was $1.16 \%$ s for 3-s motion duration. Moreover, Soyka et al. (2011) reported thresholds for linear self-motion discrimination of $0.018 \mathrm{~m} / \mathrm{s}^{2}$ for a motion duration of $2.36 \mathrm{~s}$. The mean linear self-motion threshold in the older sample of our study was $0.02 \mathrm{~m} / \mathrm{s}^{2}$.

Moreover, we found that pitch and roll rotation thresholds were lower when compared to yaw rotation thresholds. This finding is consistent with other studies (e.g., Valko et al. 2012). Two reasons can account for this finding: First, roll and pitch rotation also stimulate the otolith organs, while this is not (or much less) the case for yaw rotation stimuli. Second, roll and pitch rotations evoke a more pronounced stimulation of extravestibular sensory self-motion information, such as proprioceptive cues (see for example Mittelstaedt 1996).

Thresholds as a function of age?

While no consistent influence of gymnastic expertise on self-motion discrimination thresholds was found, there was a significant (or nearly significant) main effect of age for all motions. Can self-motion discrimination thresholds therefore be described as a function of age and consequently as a function of sensory (vestibular) development in adolescence? A potential confound of age is body height. Given that the axis of rotation was not head-centered, body size 

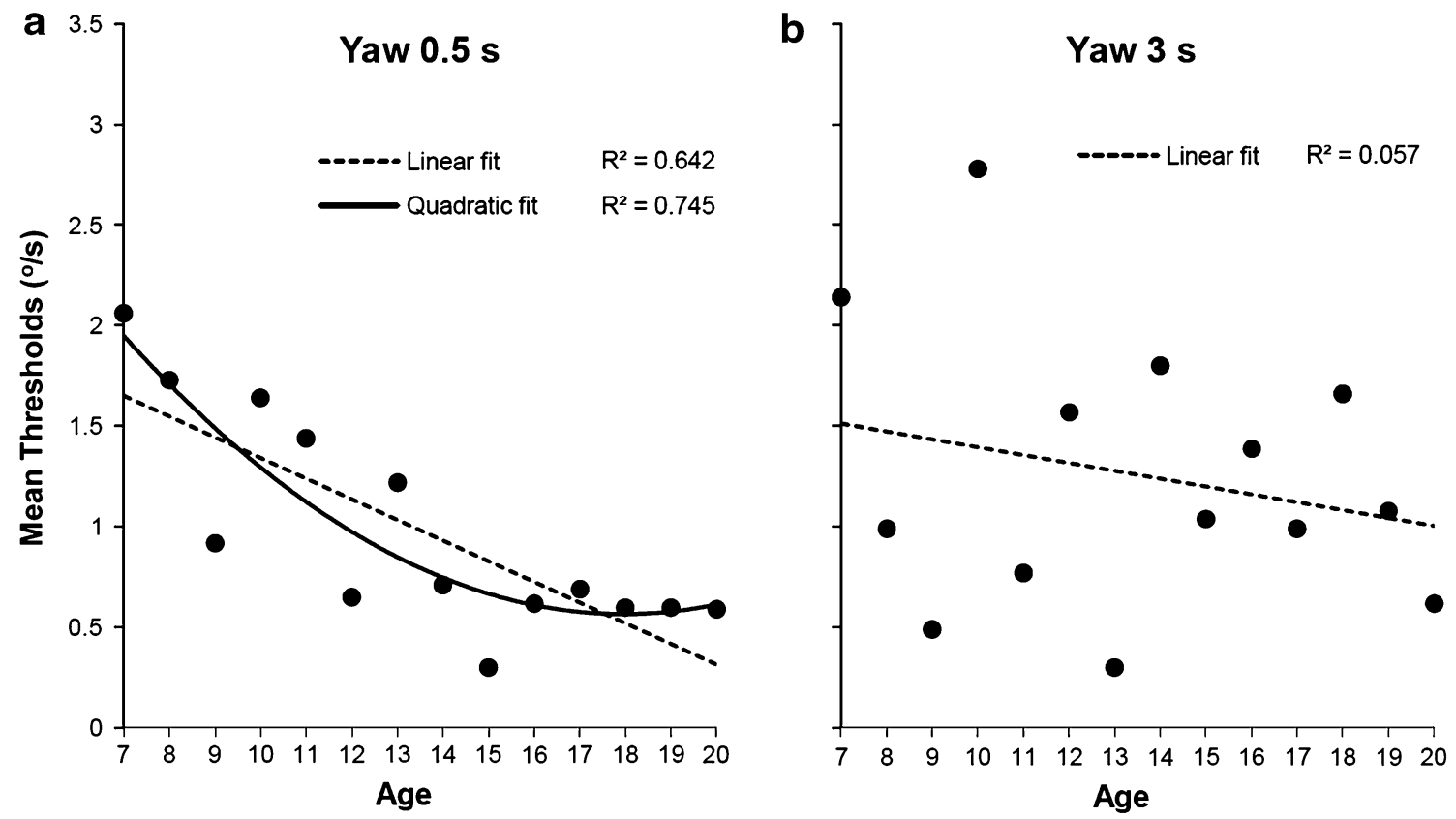

Fig. 5 Mean thresholds for $0.5 \mathrm{~s} \mathrm{(a)} \mathrm{and} 3 \mathrm{~s}$ (b) yaw rotation as a function of age. Dotted lines represent linear fits, and solid line represents quadratic fit (illustrated only for $0.5 \mathrm{~s}$ duration). Note that $R^{2}$ is based on the fit of the means for each age

may have influenced vestibular thresholds for roll and pitch rotation because greater distance of the head with respect to the axis of rotation resulted in stronger otolith stimulation. As a result, we decided not to interpret the results for roll and pitch rotations as function of age. Moreover, the main effect of age for y translation may be driven by the interaction between age and group (lower thresholds of gymnasts for the older group). However, we found effects of age for yaw rotation thresholds where tangential and centripetal forces do not depend on body height. In addition to this, yaw thresholds were not influenced by expertise. To further analyze the effect of age, we computed a regression analysis for the 0.5- and 3-s motion duration with age as predictor. Age turned out to be a significant predictor for the 0.5 -s duration yaw rotation thresholds, $\beta_{\text {standardized }}=-0.42$, $t=-3.12, p=0.003, R^{2}=0.18$ but not for the 3-s duration yaw rotation thresholds, $\beta_{\text {standardized }}=-0.016, t=-0.12$, $p=0.913, R^{2}=0.02$. The mean yaw rotation thresholds for each age and the linear fits for the 0.5- and 3-s duration are illustrated in Fig. 5a, b. For 0.5-s duration, yaw rotation thresholds seem to reach a plateau around 16 years. Thus, mean thresholds can be best described by a quadratic function (see Fig. 5a).

\section{General discussion}

The aim of this study was to compare self-motion perception thresholds of artistic gymnasts with those of controls.
We found that-at least for the older group-artistic gymnasts outperformed controls in linear leftward-rightward motion discrimination but not (or only by trend) for angular motion discrimination. Linear leftward-rightward motion is encoded by the otolith organs. This result suggests that self-motion expertise is not associated with a general increase in the sensitivity of self-motion perception but may be specifically associated with superior otolith-based vestibular processing. However, changes in body orientation with respect to gravity are processed by the otolith organs. Consequently, an advantage in the processing of otolith signals for gymnasts should also result in a better performance for roll and pitch rotation discrimination. This was not the case in our sample. An advantage of artistic gymnasts' expertise manifested itself only in the type of motion where otolith input is processed in the absence of a change in canal activity (there is also a canal signal during translation that signals zero angular motion). A further difference between linear and angular motion is the extent to which these motions evoke extravestibular cues. Given that roll and pitch rotation lead to a tilt of the entire body, these motions provide stronger somatosensory cues (e.g., in the skin surface around the gluteal region) and possibly also stronger visceral cues than translational motion. Our results suggest that gymnastic expertise is associated with more efficient processing of self-motion information when less extravestibular information is available and when the angular velocity signals from the semicircular canals cannot be used in combination with the linear acceleration 
signal from the otoliths. Balance functions require accurate perception of otolith inputs, which are immensely important for gymnastic feats. It is possible that the processing of otolith signals is an important feature for superior performance in artistic gymnastics.

Although there were no significant differences between the gymnasts and controls in the younger group, it was surprising that thresholds tended to be higher for the gymnasts than for controls in the younger group. Gymnastic training of several years in young children seems to have no effect on basic self-motion perception (i.e., direction discrimination ability). Two reasons can account for the fact that thresholds of gymnasts were lower in the older group. First, training history is longer and training intensity is higher for the older group, suggesting that extensive long-term experience in the control of self-motion can lead to more efficient processing of translational self-motion perception. However, the difference between gymnasts and controls in the older sample could also be the result of a selection effect: it is possible that the younger group included gymnasts who will not meet the necessary level of performance to become a successful athlete in the future, whereas the gymnasts in the older group have been successful in the past and perform on the highest level of expertise. Future studies are needed in order to disentangle training versus selection effects for superior self-motion perception performance.

It is also important to highlight that the artistic gymnasts' thresholds were not significantly higher than those of controls for any type of motion, ruling out a decrease in vestibular sensitivity due to repeated training (habituation). These findings are interesting with respect to previous studies that found an influence of gymnastic expertise on the VOR (Alpini et al. 2009; Quarck and Denise 2005; Tanguy et al. 2008b). These studies pointed out that the motions performed by the artistic gymnasts lead to a repeated, high-intense stimulation of the vestibular system, resulting in vestibular habituation. Our findings together with those about the VOR indicate that habituation involves the interaction of visual and vestibular information rather than the level of "purely" vestibular perception alone. This conclusion is in line with Balter et al.'s (2004) study in which no difference in vestibular response to galvanic vestibular stimulation of gymnasts was found. Note, however, that VOR changes may also reflect an adjustment of oculomotor rather than vestibular or visual-vestibular sensory processes.

In addition to expertise-related findings, we observed robust effects of duration and age for all motion thresholds. In general, velocity thresholds were lower for the shorter motion when compared to the longer motion. This finding is not new and has been reported in earlier studies (Benson et al. 1989; Grabherr et al. 2008; Soyka et al. 2011). Moreover, thresholds were lower in older when compared to younger participants. We cannot attribute these differences to age alone for roll and pitch rotations (due to the conceivable confound body height) and for $y$ translation (due to the confound expertise). However, there was an effect of age for yaw rotation thresholds where these possibly confounding factors were absent. To the best of our knowledge, this was the first report of self-motion perception threshold in children and young adults (7-20 years). Therefore, our yaw rotation data can serve as baseline for the assessment of young people showing signs of vestibular dysfunctions. In our study, only the thresholds for the shorter motion $(0.5 \mathrm{~s})$ decreased as a function of age, but not those for the longer motion (3 s), Interestingly, Roditi and Crane (2012a) found that yaw rotation discrimination thresholds for 2-s motion duration were independent of age (age range between 20 and 70 years). They also tested yaw rotation discrimination thresholds for 1-s motion duration. The thresholds for $1 \mathrm{~s}$ could potentially be described as a function of age (see Fig. 11h in Roditi and Crane 2012a). It seems to be important to consider motion duration when investigating the effects of age in self-motion perception.

Moreover, previous studies focused on the impairment in vestibular functions in the older age (e.g., Baloh et al. 1993; Maes et al. 2010; Paige 1994; Peterka et al. 1990). These studies have shown that older participants $(>50)$ have an impaired ability in self-motion discrimination (Kingma 2005; Roditi and Crane 2012a, b). For example, Roditi and Crane (2012a) found that leftward-rightward (and forward-backward) translation thresholds were significantly higher for participants over 50 years. They suggested that impaired self-motion discrimination with age was a perceptual consequence of otolith deterioration shown in physiological studies (Bergstrom 1973; Richter 1980; Rosenhall 1973). We highlight that age can have positive effects on vestibular functions in a younger sample. Our results suggest that vestibular perception (at least canal-based vestibular perception of short motions) is still improving from 7 years onwards, presumably reaching a plateau in young adulthood, and, according to Roditi and Crane (2012a) and Kingma's (2005) data, will start worsening at around 40 years. In this respect, our results are roughly in line with a recent study on balance functions with children (Hsu et al. 2009) suggesting that children reach adult balance level at the age of 12 years (see also Steindl et al. 2006, for a comparison between the development of different sensory systems in balance functions).

It remains an open question why there was no difference between artistic gymnasts and controls for the angular motions. Given that the angular motions (especially the pitch and roll) are most relevant for balance control and part of many gymnastic feats (Bechter et al. 2009), we expected the largest difference particularly for those motions. A possible explanation would be that any 
differences in self-motion perception in artistic gymnasts are specific for the trained conditions. Three major differences between the training condition and the test condition of this study can be found. First, the motions that are trained are more complex and more intense than the ones we tested in this study. Second, gymnastic performance is active and voluntary, thus involving intention, motor planning, and motor activity. Third, gymnasts perform selfmotion while they are at the same time exposed to visual input, whereas no visual input was provided in this study. The absence of an effect of expertise in untrained conditions is not unusual. For example, Asseman et al. (2004) found that the advantage of elite gymnasts in postural control in the handstand does not transfer to upright standing postures (see also Asseman et al. 2008). Furthermore, we recently found that self-motion perception thresholds decreased via perceptual training in the light, but there was no transfer to self-motion perception thresholds in the dark (Hartmann et al. 2013). The removal of light has been found to hamper performance in other tasks similarly for gymnasts and controls alike (e.g., Asseman et al. 2008; Sahli et al. 2011). These findings highlight the importance of visual input in self-motion perception. Note, however, that other studies on postural control found that expertise reduces the dependency of vision (Croix et al. 2010; Paillard and Noé 2006; Vuillerme et al. 2001). Nevertheless, it is possible that training-specific processing of vestibular information (often in the context of concurrent visual information and motor activity) is modulated and does not transfer to self-motion perception in untrained conditions (i.e., passive self-motion perception in the dark). However, we found improved performance in gymnasts for linear leftward-rightward motion, and possible explanations for this finding are outlined above (see first paragraph of discussion). Moreover, a longitudinal study would be helpful in order to disambiguate training-specific effects from selection effects.

To conclude, we provide new evidence that self-motion perception is different in artistic gymnasts when compared to controls. Interestingly, this difference was specific for the linear motion. No difference was found for angular motions that are an integral part of gymnastic motion sequences. It is therefore unlikely that superior self-motion abilities in artistic gymnasts are achieved by an overall higher sensitivity of the vestibular system. Moreover, we found an effect of age on the sensitivity of self-motion perception. Thus, functional plasticity of the vestibular system seems to be driven by normal maturation rather than expertise in selfmotion. Given that the vestibular system is involved in the control of the autonomic nervous system (e.g., heart rate and blood pressure; see for example El Sayed et al. 2012) and in other remote tasks, the limited malleability of the vestibular system due to external influences might be protective against too much variance in these networks.

Acknowledgments This study was funded by the Swiss National Science Foundation (Pro*Doc Grant PDFMP1_127238). We thank all our participants and their parents for their contribution to this study. We also thank Andrea Kienholz for her assistance in the recruitment of participants.

\section{References}

Alpini D, Botta M, Mattei V, Tornese D (2009) Figure ice skating induces vestibulo-ocular adaptation specific to required athletic skills. Sport Sci Health 5(3):129-134

Asseman F, Caron O, Crémieux J (2004) Is there a transfer of postural ability from specific to unspecific postures in elite gymnasts? Neurosci Lett 358(2):83-86

Asseman F, Caron O, Crémieux J (2008) Are there specific conditions for which expertise in gymnastics could have an effect on postural control and performance? Gait Posture 27(1):76-81

Baloh R, Jacobson K, Socotch T (1993) The effect of aging on visualvestibuloocular responses. Exp Brain Res 95(3):509-516

Balter SGT, Stokroos RJ, Akkermans E, Kingma H (2004) Habituation to galvanic vestibular stimulation for analysis of postural control abilities in gymnasts. Neurosci Lett 366(1):71-75

Bechter B, Bechter M, Haller C, Donàth V (2009) Geräte- und Kunstturnen/Trampolin Fachgrundlagen. Bundesamt für Sport BASPO, Magglingen

Benson AJ, Spencer MB, Stott JR (1986) Thresholds for the detection of the direction of whole-body, linear movement in the horizontal plane. Aviat Space Environ Med 57(11):1088-1096

Benson AJ, Hutt EC, Brown SF (1989) Thresholds for the perception of whole body angular movement about a vertical axis. Aviat Space Environ Med 60(3):205-213

Bergstrom B (1973) Morphology of the vestibular nerve II: the number of myelinated vestibular nerve fibers in man at various ages. Acta Otolaryngol 76:173-179

Berry DA (1987) Logarithmic transformations in ANOVA. Biometrics 43:439-456

Bertolini G, Ramat S, Bockisch CJ, Marti S, Straumann D, Palla A (2012) Is vestibular self-motion perception controlled by the velocity storage? Insights from patients with chronic degeneration of the vestibulo-cerebellum. PLoS ONE 7(6):e36763

Blair S, Gavin M (1979) Response of the vestibulo-ocular reflex to differing programs of acceleration. Invest Ophthalmol Vis Sci 18(10):1086-1090

Bringoux L, Marin L, Nougier V, Barraud PA, Raphel C (2000) Effects of gymnastics expertise on the perception of body orientation in the pitch dimension. J Vestib Res 10(6):251-258

Carrick FR, Oggero E, Pagnacco G, Brock JB, Arikan T (2007) Posturographic testing and motor learning predictability in gymnasts. Disabil Rehabil 29(24):1881-1889

Clément G, Tilikete C, Courjon JH (2008) Retention of habituation of vestibulo-ocular reflex and sensation of rotation in humans. Exp Brain Res 190(3):307-315

Croix G, Chollet D, Thouvarecq R (2010) Effect of expertise level on the perceptual characteristics of gymnasts. J Strength Cond Res 24(6): 1458

El Sayed K, Dawood T, Hammam E, Macefield VG (2012) Evidence from bilateral recordings of sympathetic nerve activity for lateralisation of vestibular contributions to cardiovascular control. Exp Brain Res 221(4):427-436. doi:10.1007/s00221-012-3185-6 
Gautier G, Thouvarecq R, Larue J (2008) Influence of experience on postural control: effect of expertise in gymnastics. J Mot Behav 40(5):400-408

Grabherr L, Nicoucar K, Mast FW, Merfeld DM (2008) Vestibular thresholds for yaw rotation about an earth-vertical axis as a function of frequency. Exp Brain Res 186(4):677-681. doi:10.1007/ s00221-008-1350-8

Hartmann M, Grabherr L, Mast FW (2012) Moving along the mental number line: interactions between whole-body motion and numerical cognition. J Exp Psychol Hum Percept Perform 38(6):1416-1427. doi:10.1037/a0026706

Hartmann M, Furrer S, Herzog MH, Merfeld DM, Mast F (2013) Self-motion perception training: thresholds improve in the light but not in the dark. Exp Brain Res 226(2):231-240

Hrysomallis C (2011) Balance ability and athletic performance. Sports Med 41(3):221-232

Hsu YS, Kuan CC, Young YH (2009) Assessing the development of balance function in children using stabilometry. Int J Pediatr Otorhinolaryngol 73(5):737-740

Jäger J, Henn V (1981) Habituation of the vestibulo-ocular reflex (VOR) in the monkey during sinusoidal rotation in the dark. Exp Brain Res 41(2):108-114

Keene ON (2007) The log transformation is special. Stat Med 14(8):811-819

Kingma H (2005) Thresholds for perception of direction of linear acceleration as a possible evaluation of the otolith function. BMC Ear Nose Throat Disord 5(1):5

Lee MY, Kim MS, Park BR (2004) Adaptation of the horizontal vestibuloocular reflex in pilots. Laryngoscope 114(5):897-902. doi:10.1097/00005537-200405000-00021

Leek MR (2001) Adaptive procedures in psychophysical research. Percept Psychophys 63(8):1279-1292

Louer LEAM, Elferink-Gemser MT, Visscher C (2012) The perfect elite gymnast, does he exist? A systematic review. Ann Res Sport Phys Act 3:39-61

MacNeilage PR, Banks MS, DeAngelis GC, Angelaki DE (2010) Vestibular heading discrimination and sensitivity to linear acceleration in head and world coordinates. J Neurosci 30(27):9084-9094

Maes L, Dhooge I, D'haenens W, Bockstael A, Keppler H, Philips B, Swinnen F, Vinck BM (2010) The effect of age on the sinusoidal harmonic acceleration test, pseudorandom rotation test, velocity step test, caloric test, and vestibular-evoked myogenic potential test. Ear Hearing 31(1):84

Merfeld DM (2012) Spatial orientation and the vestibular system. In: Wolfe JM, Kluender KR, Levi DM (eds) Sensation and perception, 3rd edn. Sinauer Associates Inc, Massachusetts

Mittelstaedt H (1996) Somatic graviception. Biol Psychol 42(1-2):53-74
Osterhammel P, Terkildsen K, Zilstorff K (1968) Vestibular habituation in ballet dancers. Acta Otolaryngol 66(3):221-228

Paige GD (1994) Senescence of human visual-vestibular interactions: smooth pursuit, optokinetic, and vestibular control of eye movements with aging. Exp Brain Res 98(2):355-372

Paillard T, Noé F (2006) Effect of expertise and visual contribution on postural control in soccer. Scand J Med Sci Sports 16(5):345-348

Peterka R, Black FO, Schoenhoff M (1990) Age-related changes in human vestibulo-ocular reflexes: sinusoidal rotation and caloric tests. J Vestibul Res 1:49-59

Quarck G, Denise P (2005) Caractéristiques du reflexe vestibulo-oculaire chez les gymnastes. Science et motricité 2:101-112

Richter E (1980) Quantitative study of human scarpa's ganglion and vestibular sensory epithelia. Acta Otolaryngol 90:199-208

Roditi RE, Crane BT (2012a) Directional asymmetries and age effects in human self-motion perception. J Assoc Res Otolaryngol 13:381-401

Roditi RE, Crane BT (2012b) Suprathreshold asymmetries in human motion perception. Exp Brain Res 219:369-379

Rosenhall U (1973) Degenerative patterns in the aging human vestibular neuro-epithelia. Acta Otolaryngol 76:208-220

Sahli S, Ghroubi S, Rebai H, Chaâbane M, Yahia A, Pérennou D, Elleuch M (2011) The effect of circus activity training on postural control of 5-6-year-old children. Sci Sports 28:11-16

Soyka F, Robuffo Giordano P, Beykirch K, Bulthoff HH (2011) Predicting direction detection thresholds for arbitrary translational acceleration profiles in the horizontal plane. Exp Brain Res 209(1):95-107. doi:10.1007/s00221-010-2523-9

Steindl R, Kunz K, Schrott-Fischer A, Scholtz A (2006) Effect of age and sex on maturation of sensory systems and balance control. Dev Med Child Neurol 48(6):477-482

Tanguy S, Quarck G, Etard O, Gauthier A, Denise P (2008a) Are otolithic inputs interpreted better in figure skaters? Neuro Rep 19(5):565-568

Tanguy S, Quarck G, Etard O, Gauthier A, Denise P (2008b) Vestibulo-ocular reflex and motion sickness in figure skaters. Eur $\mathrm{J}$ Appl Physiol 104(6):1031-1037

Taylor MM, Creelman CD (1967) Pest-efficient estimates on probability functions. J Acoust Soc Am 41(4):782

Tschiassny K (1957) Studies concerning vestibular factors in the ballet dancer, the pigeon, and the blind person. Trans Am Acad Ophthalmol Otolaryngol 61(4):503-506

Valko Y, Lewis RF, Priesol AJ, Merfeld DM (2012) Vestibular labyrinth contributions to human whole-body motion discrimination. J Neurosci 32(39):13537-13542

Vuillerme N, Danion F, Marin L, Boyadjian A, Prieur J, Weise I, Nougier V (2001) The effect of expertise in gymnastics on postural control. Neurosci Lett 303(2):83-86 